\title{
Beat frequency of cilia from sites of purulent infection
}

\author{
R WILSON, DA SYKES, D CURRIE, PJ COLE \\ From the Host Defence Unit, Department of Thoracic Medicine, Cardiothoracic Institute, London
}

\begin{abstract}
Mucociliary clearance depends on the interaction between cilia and mucus; it is delayed in the presence of purulent secretions. Nasal mucociliary clearance was examined by the saccharin method and nasal ciliary beat frequency by a photometric technique. Four groups were studied: normal controls, patients with bronchiectasis without nasal symptoms, patients with chronic mucopurulent sinusitis alone, and patients with chronic mucopurulent sinusitis and bronchiectasis. Nasal mucociliary clearance was prolonged in infected patients. Cilia obtained from the site of purulent secretions were found to beat more slowly in vitro (mucopurulent sinusitis $12.1 \mathrm{~Hz}$, mucopurulent sinusitis and bronchiectasis $11.6 \mathrm{~Hz}$ ), than those obtained from normal controls $(14.3 \mathrm{~Hz})$ and from patients with bronchiectasis alone $(13.6 \mathrm{~Hz})$. The cause of the ciliary slowing seemed most likely to be the release of host factors during the inflammatory response, rather than the particular organism isolated. Ciliary slowing may contribute to the observed delay of mucociliary clearance in conditions in which purulent secretions are present.
\end{abstract}

Mucociliary clearance has been shown to be delayed in conditions in which purulent secretions are common: Lourenço et al $^{1}$ found slower clearance in bronchiectatic patients than in normal controls, Kollberg $e t a l^{2}$ and Wood $e t a l^{3}$ found delayed clearance in patients with cystic fibrosis, and Stanley et al found delayed clearance in mucopurulent sinusitis. ${ }^{4}$

This delayed clearance is likely to be multifactorial. The mucociliary system depends on an interaction between cilia and mucus. The properties of mucus may change from low viscosity and high elastic recoil, which are ideal for mucociliary transport. ${ }^{5}$ Burgi $^{6}$ showed that infected mucus has an increased viscosity that is mainly due to increased DNA fibre content. There may be loss of ciliated epithelium. Reimer $\mathrm{et} \mathrm{al}^{7}$ studied biopsy specimens of maxillary sinus mucosa, with scanning electron microscopy, in patients with chronic mucopurulent sinusitis. Large areas of unciliated epithelium were seen.

The present study was designed to investigate the beating of cilia at sites of purulent infection.

\section{Methods}

\section{PATIENTS}

Four groups were studied. These comprised: 10 normal controls (five male and five female, aged 20-59

Address for reprint requests: Dr $\mathbf{R}$ Wilson, Host Defence Unit, Cardiothoracic Institute, Brompton Hospital, London SW3 6HP.

Accepted 6 December 1985 years) with no past history of nasal or pulmonary disease; 10 patients with chronic mucopurulent sinusitis (three male and seven female, aged 12-75 years) but without any chest symptoms; 10 patients with bronchiectasis but no nasal symptoms (six male and four female, aged 16-60 years); and 10 patients with bronchiectasis and mucopurulent sinusitis (five male, five female; aged 16 to 44). Mucopurulent sinusitis was diagnosed by history, examination, and the appearance of sinus radiographs. Bronchiectasis was diagnosed by history, examination, the appearance of chest radiographs, and additional bronchographic information in nine patients. Only patients with purulent anterior nasal secretions on rhinoscopy were included. Serum immunoglobulin deficiency was excluded in all patients. No patient or normal control had received antibiotics or gave a history of viral infection in the four weeks before the study.

\section{MEASUREMENT OF NASAL MUCOCILIARY CLEARANCE AND CILIARY BEAT FREQUENCY} Nasal mucociliary clearance was determined in an unobstructed nostril with the saccharin test. ${ }^{8}$ The time from placement of a $1 \mathrm{~mm}$ diameter particle of saccharin on the inferior nasal turbinate, $1 \mathrm{~cm}$ from its anterior end, to the subject's first experience of a sweet taste was recorded in minutes. The subject was positioned with head slightly forward and requested not to sniff, sneeze, cough, eat, or drink during the test. The test was terminated after 60 minutes and those subjects not tasting had their ability to taste 
saccharin verified by placing a particle on the tongue. In vitro examination of ciliary beat frequency was performed by a photometric technique. ${ }^{9-11}$ Cilia were obtained from the inferior turbinate of the same nostril without local anaesthesia by a brushing technique, ${ }^{9}$ the strips of ciliated epithelium being dislodged by brisk agitation of the cytology brush in $2 \mathrm{ml}$ of medium 199 cell culture fluid (Flow laboratories). The suspension of ciliated epithelium was immediately transferred to a sealed microscope coverslip slide preparation with a pipette, and the preparation placed on an electronically controlled warmstage (Microtec) at $37^{\circ} \mathrm{C}$, and allowed to equilibrate for 15 minutes. During this time strips of ciliated epithelium were identified on the slide and their positions marked. At least six strips of epithelium were identified and usually 10 , single ciliated cells or small groups of ciliated cells being ignored as ciliary beat frequency measured at such sites is often inaccurate. If six strips of epithelium could not be identified a second nasal brushing was obtained from the same nostril.

For the measurement of ciliary beat frequency cilia were viewed directly on a Leitz Dialux 20 phase contrast microscope at magnification $\times 320$ by bright field illumination. This allowed an assessment of their beating pattern to be made. The cilia were positioned to interrupt the passage of light through a small diaphragm into a Leitz MPV compact microscope photometer, which converted light intensity into an electrical signal; this was subsequently converted into a reading of ciliary beat frequency $(\mathrm{Hz})$ by a ciliary beat frequency processor unit. ${ }^{11}$ Ten readings of ciliary beat frequency were made using each ciliated epithelial strip identified, and the mean was calculated.

\section{BACTERIOLOGY}

Cultures were set up from a swab of the anterior nares and from a sample of the medium 199 cell culture fluid containing ciliated epithelium. Standard and selective bacteriological plates ${ }^{10}$ were incubated aerobically and anaerobically overnight at $37^{\circ} \mathrm{C}$.

\section{OTHER STUDIES}

The following studies were performed only on selected subjects.

Transmission electron microscopy of cilia obtained by brushing the inferior turbinate of three patients with a ciliary beat frequency less than $11 \mathrm{~Hz}(10.3,9.8$, $9.8 \mathrm{~Hz}$ ) was performed. The brush was agitated in cacodylate buffered $2.5 \%$ glutaraldehyde and postfixed in $1 \%$ osmium tetroxide. After rinsing, the brushings of ciliated epithelium were embedded in a drop of $2 \%$ liquid agar and gently centrifuged. The agar was allowed to set, then processed through to embedding in Araldite as routine for tissues. Semithin sections were cut and stained in $1 \%$ toluidine blue for $\overrightarrow{\vec{F}}$ light microscopy and suitable areas selected and trimmed for ultrathin sectioning. These were stained $\frac{\bar{C}}{O}$ in uranyl acetate and lead nitrate for transmission $\overline{\bar{n}}$ electron microscopy.

Treatment In two patients (ciliary beat frequency $9.8 \mathrm{~Hz}$ for both) measurements of nasal mucociliaryes clearance and ciliary beat frequency were made after $\vec{\circ}$ treatment appropriate to the isolated organism. $\overrightarrow{.}$ Treatment was begun after the first brushing. Topical $\stackrel{\omega}{\omega}$ nasal treatment continued up to the time of the follow up brushings. Betamethasone with neomycin nose drops have been shown to inhibit ciliary beating in.vitro but not to affect adversely the ciliary beat frequency in vivo. ${ }^{12}$

Serial tests (1) A single normal volunteer underwento measurement of nasal mucociliary clearance and ${ }_{-}^{\supset}$ nasal ciliary beat frequency on 10 occasions on $\vec{c}$ different days. This allowed assessment of the within subject variability of these tests. (2) Three patients ${ }_{-}^{\circ}$ with bronchiectasis and mucopurulent sinusitis each $\ddot{\infty}_{\infty}$ underwent measurement of nasal ciliary beat fre-. quency on three successive days. On one of the days the ciliary beat frequency of the epithelium on the slide was measured after two and four hours at $37^{\circ} \mathrm{C}$.

STATISTICS

The mean ciliary beat frequency of each group was $\overrightarrow{\overrightarrow{\vec{O}}}$ compared with the unpaired Student's $t$ test (183 degrees of freedom). The mean nasal mucociliary? clearance of each group was compared with the same test, 60 being taken as the value for any subject who had not tasted saccharin after 60 minutes.

\section{Results}

Nasal mucociliary clearance (fig 1) was significantlyo̊ slower in patients with mucopurulent sinusitis than in normal controls $(p<0.001)$, and in patients with의 bronchiectasis and mucopurulent sinusitis than in patients with bronchiectasis alone $(p<0.001)$. There was also a significant difference between the nasal on mucociliary clearance of normal controls and patients with bronchiectasis alone $(\mathrm{p}<0.02)$.

The mean ciliary beat frequency (fig 2) was $\omega$ significantly slower in patients with mucopurulent sinusitis (12.1) than in normal controls (14.3) $(\mathrm{p}<0.001)$, and in those with bronchiectasis with mucopurulent sinusitis (11.6) than in those with bron-? chiectasis alone $(13.6)(p<0.001)$. There was no $\frac{T}{C}$ significant difference between the mean ciliary beat frequency of normal controls and of patients with bronchiectasis alone. As only 10 subjects were stud- $\stackrel{\mathbb{Q}}{\complement}$ ied, however, only a gross difference would have been detected in this study.

The bacteriological results are shown in table ? 


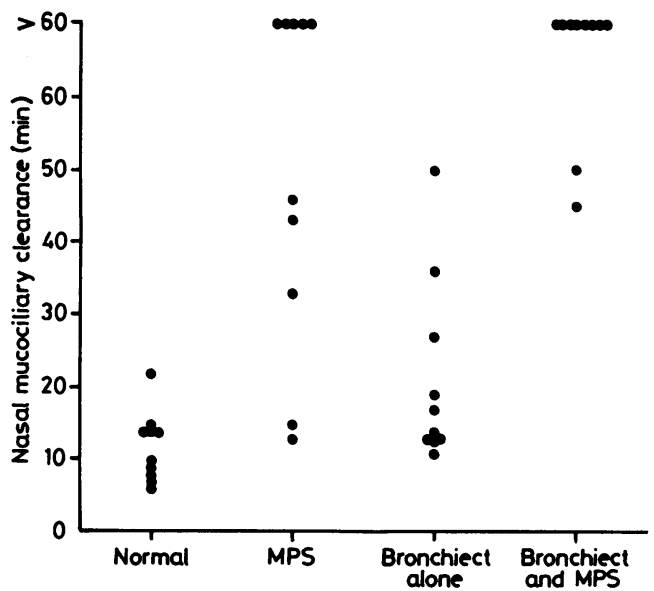

Fig 1 Nasal mucociliary clearance measured by the saccharin method in four groups of patients.

MPS-mucopurulent sinusitis; Bronchiect-bronchiectasis.

Table 1 Bacteria isolated from culture of an anterior nasal swab and from a sample of the nasal brushing into medium 199

\begin{tabular}{lll}
\hline Group & Bacteria & $\begin{array}{l}\text { No of } \\
\text { isolates }\end{array}$ \\
\hline Normal controls & $\begin{array}{l}\text { No growth } \\
\text { Staphylococcus aureus }\end{array}$ & 6 \\
Patients with bronchiectasis \\
alone & Staphylococcus epidermidis & 2 \\
& No growth & 8 \\
Patients with mucopurulent & Staphylococcus aureus & 2 \\
sinusitis & No growth & 2 \\
& $\begin{array}{l}\text { Staphylococcus aureus } \\
\text { Pseudomonas aeruginosa }\end{array}$ & 4 \\
& $\begin{array}{l}\text { Streptococcus pneumoniae } \\
\text { Staphylococcus epidermidis }\end{array}$ & 1 \\
Patients with bronchiectasis & Coliform & 1 \\
and mucopurulent sinusitis & No growth & 2 \\
& $\begin{array}{l}\text { Staphylococcus aureus } \\
\text { Pseudomonas aeruginosa }\end{array}$ & 3 \\
& $\begin{array}{l}\text { Staphylococcus epidermidis } \\
\text { Proteus vulgaris }\end{array}$ & 2 \\
\hline
\end{tabular}

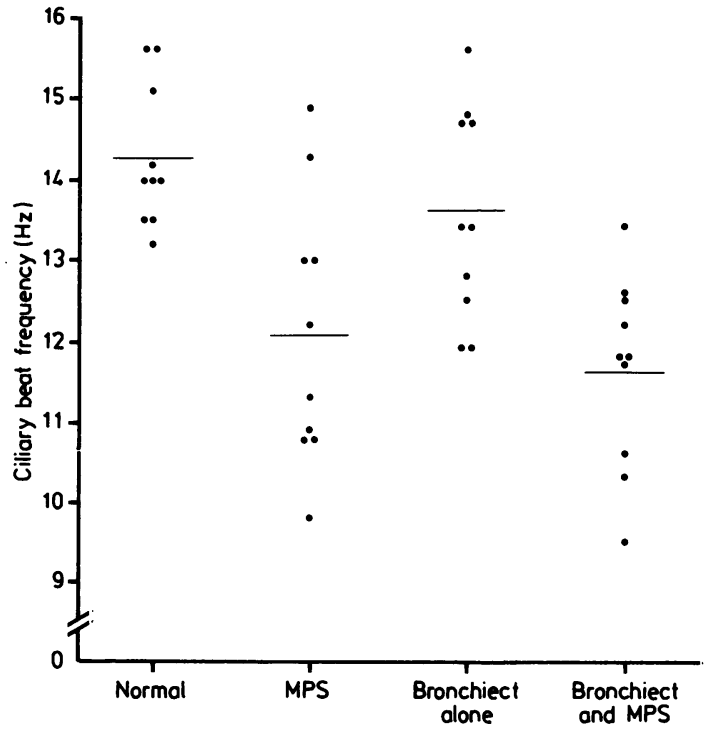

Fig 2 Ciliary beat frequency measured by a photometric technique in four groups of patients. MPS-mucopurulent sinusitis; Bronchiect-bronchiectasis.

Staphylococcus aureus was the most frequent isolate, and as expected positive cultures were more frequent in the patients with purulent secretions.

The beating pattern of the cilia was normal in each case, with the exception of one patient whose nasal swab and brushing grew Pseudomonas aeruginosa. In this case the cilia appeared dyskinetic (loss of the usual coordinated beating pattern), but they returned to normal after clearance of the organism with antibiotic treatment. Static cilia were not observed in any specimen obtained.

No dynein arm defects were observed in the three specimens examined by transmission electron microscopy. An increased frequency of compound cilia was seen in one patient (eight of 179 cilia examined) and absence of some central microtubules observed in another.

With treatment (table 2) the infecting organism was

Table 2 Effect of treatment on ciliary beat frequency $(C B F)$ and nasal mucociliary clearance (NMCC) in patients with mucopurulent sinusitis

\begin{tabular}{|c|c|c|c|c|}
\hline & & \multicolumn{3}{|c|}{ Duration of treatment } \\
\hline & & At diagnosis & 6 weeks & 12 weeks \\
\hline $\begin{array}{l}\text { Patient } 1^{*} \text { (Staphylococcus aureus) } \\
\text { Patient } 2 \dagger \text { (Pseudomonas aeruginosa) }\end{array}$ & $\begin{array}{l}\text { CBF (Hz) } \\
\text { NMCC (min) } \\
\text { CBF (Hz) } \\
\text { NMCC (min) }\end{array}$ & $\begin{array}{l}9.8 \\
33 \\
9.8 \\
>60\end{array}$ & $\begin{array}{l}\frac{10.8}{11.8} \\
35\end{array}$ & $\begin{array}{l}12.1 \\
30 \\
-\end{array}$ \\
\hline
\end{tabular}

*Treatment: betamethasone with neomycin nose drops.

†Treatment: intravenous antibiotics followed by betamethasone with neomycin nose drops, gentamicin with hydrocortisone nose drops. 
Table 3 Variability of ciliary beat frequency (CBF) measured on three successive days in three patients with mucopurulent sinusitis

\begin{tabular}{|c|c|c|c|c|c|c|c|}
\hline \multirow[t]{2}{*}{ Patient no } & \multirow[t]{2}{*}{ Bacteria isolated } & \multirow{2}{*}{$\begin{array}{l}N M C C \\
(\min )\end{array}$} & \multicolumn{5}{|c|}{ Ciliary Beat Frequency $(\mathrm{Hz})$} \\
\hline & & & Day 1 & Day 2 & Day 3 & After $2 h^{*}$ & After $4 h^{*}$ \\
\hline $\begin{array}{l}1 \\
2 \\
3\end{array}$ & $\begin{array}{l}\text { Staphylococcus aureus } \\
\text { Neisseria sp Bacillus sp } \\
\text { Staphylococcus aureus }\end{array}$ & 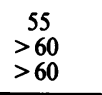 & $\begin{array}{l}11.8 \\
10.7 \\
10.3\end{array}$ & $\begin{array}{l}11.5 \\
11.2 \\
10.1\end{array}$ & $\begin{array}{l}12.0 \\
10.9 \\
10.3\end{array}$ & $\begin{array}{l}11.1 \\
12.1 \\
10.8\end{array}$ & $\begin{array}{l}11.3 \\
13.5 \dagger \\
10.9\end{array}$ \\
\hline
\end{tabular}

eradicated in both cases. Ciliary beat frequency improved $(p<0.001)$, and in one case there was an improvement in nasal mucociliary clearance.

In the volunteer subjects, the mean of 10 nasal mucociliary clearance values was 15 (range 11-25) minutes, coefficient of variation $27 \%$. The mean ciliary beat frequency for 10 separate nasal brushings (each itself a mean of 10 readings) was 14.2 (range $13.0-16.2) \mathrm{Hz}$, coefficient of variation $8 \%$.

In the three patients with mucopurulent sinusitis who each had ciliary beat frequency measured on three successive days (table 3 ) there was no significant within patient variation in ciliary beat frequency. Over four hours the frequency did not change significantly in two patients, but it increased $(p<0.001)$ in one patient.

\section{Discussion}

Several authors have suggested that slowed ciliary beating may contribute to the delay observed in mucociliary clearance in the presence of purulent secretions. Rutland and Cole $^{13}$ examined the nasal ciliary beat frequency of 10 patients with bronchiectasis, and showed that the frequency was about $10 \%$ less than in normal controls. No attempt, however, was made in this study to correlate changes with the patients' symptoms, signs, or infecting organisms. Pedersen and Nielsen ${ }^{14}$ performed a similar study in patients with cystic fibrosis, and found a greater reduction in ciliary beat frequency (to about $25 \%$ of the control value), although these specimens were examined at room temperature, not at $37^{\circ}$ as in our study and that of Rutland and Cole, ${ }^{13}$ making direct comparison between the studies difficult. Pedersen and Nielsen ${ }^{14}$ also found that the ciliary beat frequency of patients improved after treatment with antibiotics aimed at eradication of Pseudomonas aeruginosa.

Smallman et al ${ }^{15}$ showed that sputum sol phase, obtained from bronchiectatic sputum after rapid centrifugation, slowed the beating of normal human nasal cilia in vitro. This effect was neutralised by $\alpha_{1}$ antitrypsin, suggesting that the effect was due to a serine protease, possibly elastase released from neutrophils during the inflammatory response. $\doteq$ Wilson et $\mathrm{l}^{10}$ showed in a similar in vitro system that products of $P$ aeruginosa and Haemophilus influenzae $s$ may slow and disorganise normal human nasal ciliary beating, although products of Staphylococcus aureus did not do so.

In this study we have confirmed the finding of Stanley $\mathrm{et}_{\mathrm{al}}{ }^{4}$ that nasal mucociliary clearance is delayed in ${ }_{-}^{\overparen{D}}$ the presence of mucopurulent sinusitis. The ciliary $\vec{\otimes}$ beat frequency results would suggest that this is partlyo due to slowed ciliary beating. The contribution of other factors, however, such as change in mucus volume or flow characteristics and loss of ciliated epithelium, is suggested by an analysis of the nasalo mucociliary clearance compared with the ciliary beat $\stackrel{\Phi}{\Phi}$ frequency within the two purulent secretion groups. $\stackrel{2}{\vec{F}}$ In fifteen patients the nasal mucociliary clearance $\frac{3}{3}$ time was over 60 minutes, with a mean ciliary beat $\supset$ frequency of 11.7 (SD 1.4) $\mathrm{Hz}$; while in eight patients it was less than 60 minutes, with a mean ciliary beat frequency of $11.8(1.4) \mathrm{Hz}$. The findings of Rutlando et al, ${ }^{16}$ that nasal ciliary beat frequency is closely correlated with the beat frequency of intrathoracic cilia, ${ }_{-}$ would suggest that similar slowing may occur within the chest in the presence of purulent secretions.

The cause of the slowed ciliary beating cannot be 3 determined from this study. $S$ aureus was the mosto frequently isolated organism, and products of this $>$ organism did not affect human nasal ciliary beato frequency in vitro. ${ }^{10}$ The nasal mucociliary clearance and ciliary beat frequency of control subjects was not different when $S$ aureus was isolated. Stockley et a 0 have shown that the purulent appearance of sputum $N_{\omega}^{N}$ was closely related to its elastase content, ${ }^{17}$ and our infected patients had purulent anterior nasal secreeo tions. Perhaps therefore factors released during the host response-for example, neutrophil elastase-are? of greater importance than the bacterial products in the observed slowing of nasal ciliary beat frequency.

The coefficient of variation of ciliary beato frequency measured on 10 occasions in a norma $\mathbb{D}$ volunteer was small $(8 \%)$. We did not consider ito reasonable to repeat the investigation on so many occasions in a patient. In three patients who each ha\& 
repeated brushings on three successive days, however, the ciliary beat frequency did not differ significantly (table 3). The technique used requires the "washing" of ciliated epithelium in medium 199 cell culture fluid when it is being dislodged from the cytology brush before transfer to the sealed microscope coverslip slide preparation. This may remove or dilute inhibitory factors present in vivo, perhaps leading to an increase in ciliary beat frequency. To minimise this effect only $2 \mathrm{ml}$ of medium 199 were used and the ciliary beat frequency was measured immediately after 15 minutes' equilibration at $37^{\circ} \mathrm{C}$. Smallman et $\mathrm{al}^{15}$ showed that in the presence of purulent sputum sol normal ciliary beat frequency continues to fall, and Wilson et $a^{10}$ have shown that in the presence of bacteria that produce cilioinhibitory factors normal ciliary beat frequency similarly falls progressively. It would therefore be difficult to interpret changes in ciliary beat frequency over longer periods as the frequency would be predicted to increase or decrease according to the presence or absence of these factors (table 3).

The very low ciliary beat frequencies obtained in some patients raises the possibility of primary ciliary dyskinesia. Static cilia, however, were not observed; dyskinesia was observed only in the one patient with $P$ aeruginosa, and the electron microscopic findings in three patients with a very slow ciliary beat frequency were not those associated with primary ciliary dyskinesia but were those found in the presence of infection. ${ }^{18}$ Treatment leading to improvement in symptoms and eradication of the infecting organism led to a significant improvement in ciliary beat frequency in two patients (table 2).

In the patient with the lowest ciliary beat frequency (the only patient to show ciliary dyskinesia) $P$ aeruginosa was isolated from the nasal swab and brushing culture. Wilson et al ${ }^{10}$ have shown that products of this organism can slow and disorganise normal human ciliary beating in vitro. Eradication of the organism and clearing of symptoms and signs led to increase in ciliary beat frequency, return to a normal beating pattern and improvement of nasal mucociliary clearance. In two other patients from whom $P$ aeruginosa was cultured, however, the frequency was not dramatically slowed $(13.0$ and $13.4 \mathrm{~Hz})$; thus if products of this organism are important in causing ciliary dyskinesia in vivo they are not produced by all strains.

Nasal mucociliary clearance was also significantly longer in patients with bronchiectasis without nasal symptoms or abnormality on rhinoscopy (mean $20 \mathrm{~min}$ ) than in normal controls (mean $12 \mathrm{~min}$ ), although the ciliary beat frequency of these two groups did not differ. As only 10 patients were studied in each group this study would detect only gross differences in beat frequency between groups. It would anyway seem likely that the previously reported slowing of ciliary beat frequency in bronchiectasis and cystic fibrosis is anyway likely to be a phenomenon secondary to the presence of purulent secretions, and not due to a primary ciliary defect.

We would conclude from this study that nasal cilia obtained from sites of purulent secretions beat more slowly in vitro. This may contribute to the observed delay of mucociliary clearance in conditions such as bronchiectasis and mucopurulent sinusitis in which purulent secretions are present.

We would like to thank Miss Elizabeth Higgs for the bacteriology and Mr Andrew Rutman for the transmission electron microscopy. RW is supported by Janssen Pharmaceuticals, DS by Fisons, and DC by the Chest, Heart, and Stroke Association. This work is supported by the National Fund for Research into Crippling Diseases.

\section{References}

1 Lourenço RV, Loddenkemper R, Carton RW. Patterns of distribution and clearance of aerosols in patients with bronchiectasis. Am Rev Respir Dis 1972; 106:857-66.

2 Kollberg H, Mossberg B, Afzelius BA, Philipson K, Camner P. Cystic fibrosis compared with the immotilecilia syndrome. Scand J Respir Dis 1978;59:297-306.

3 Wood RE, Warner A, Hirsch J, Farrell PM. Tracheal mocociliary transport in patients with cystic fibrosis and its stimulation by terbutaline. Am Rev Respir Dis 1975; 111:733-8.

4 Stanley PJ, Wilson R, Greenstone MA, Mackay IS, Cole PJ. Abnormal nasal mucociliary clearance in patients with rhinitis and its relationship to concomitant chest disease. Br J Dis Chest 1985;79:77-82.

5 Dulfano MJ, Adler KB. Physical properties of sputum. VII. Rheologic properties and mucociliary transport. $\mathrm{Am}$ Rev Respir Dis 1975;112:341-7.

6 Burgi H. Fibre systems in sputum. Bull Physiopath Resp 1973;9:191-6.

7 Reimer A, von Mecklenburg C, Toremalm NG. The mucociliary activity of the upper respiratory tract. III. A functional and morphological study on human and animal material with special reference to maxillary sinus disease. Acta Otolaryngol (Stockh) 1978;355, suppl:1-20.

8 Stanley PJ, MacWilliam L, Greenstone MA, Mackay IS, Cole PJ. Efficacy of a saccharin test for screening to detect abnormal mucociliary clearance. $\mathrm{Br} J$ Dis Chest 1984;78:62-5.

9 Rutland J, Cole PJ. Non-invasive sampling of nasal cilia for measurement of beat frequency and study of ultrastructure. Lancet 1980;ii:564.

10 Wilson R, Roberts D, Cole PJ. Effect of bacterial products on human ciliary function in vitro. Thorax 1985; 40:125-31.

11 Greenstone MA, Logan-Sinclair R, Cole PJ. An 
automated method of recording ciliary beat frequency. IRCS Med Sci 1984;12:715-6.

12 Stanley PJ, Griffin WM, Wilson R, Greenstone MA Mackay IS, Cole PJ. Effect of betamethasone and betamethasone with neomycin nasal drops on human nasal mucociliary clearance and ciliary beat frequency. Thorax 1985;40:607-12.

13 Rutland J, Cole PJ. Nasal mucociliary clearance and ciliary beat frequency in cystic fibrosis compared with sinusitis and bronchiectasis. Thorax 1981;36:654-8.

14 Pedersen M, Nielsen OH. Ciliary motility in cystic fibrosis. In: Proceedings of 11th annual meeting of European working group on cystic fibrosis. Brussels: Belgium Cystic Fibrosis Association, 1982:108-9.
15 Smallman LA, Hill SL, Stockley RA. Reduction of ciliary beat frequencies in vitro by sputum from patients with bronchiectasis: a serine proteinase effect. Thorax 1984;39:663-7.

16 Rutland J, Griffin WM, Cole PJ. Human ciliary beat frequency in epithelium from intra- and extra-thoracic airways. Am Rev Respir Dis 1982;125:100-5.

17 Stockley RA, Hill SL, Morrison HM, Starkie CM. Elastolytic activity of sputum and its relation to purulence and to lung function in patients with bronchiectasis. Thorax 1984;39:408-13.

18 Afzelius BA. "Immotile-cilia" syndrome and ciliary abnormalities induced by infection and injury. Am Rev Respir Dis 1981;124:107-9. 